\title{
A Feed-Forward Measurement Scheme for Periodic Noise Suppression in Atomic Magnetometry
}

\author{
Carolyn O'Dwyer, ${ }^{1, \text { a) }}$ Stuart J. Ingleby, ${ }^{1}$ lain C. Chalmers, ${ }^{1}$ Paul F. Griffin, ${ }^{1}$ and Erling Riis ${ }^{1}$ \\ Department of Physics, SUPA, University of Strathclyde, Glasgow, G4 ONG, UK
}

(Dated: 15 March 2020)

\begin{abstract}
We present an unshielded, double-resonance magnetometer in which we have implemented a feedforward measurement scheme in order to suppress periodic magnetic noise arising from, and correlated with, the mains electricity alternating current (AC) line. The technique described here uses a single sensor to track ambient periodic noise and feed forward to suppress it in a subsequent measurement. This feed forward technique has shown significant noise suppression of electrical mains-noise features of up to $22 \mathrm{~dB}$ under the fundamental peak at $50 \mathrm{~Hz}, 3 \mathrm{~dB}$ at the first harmonic $(100 \mathrm{~Hz})$, and $21 \mathrm{~dB}$ at the second harmonic $(150 \mathrm{~Hz})$. This technique is software based, requires no additional hardware, and is easy to implement in an existing magnetometer.
\end{abstract}

\section{INTRODUCTION}

Unshielded magnetic sensors that operate at geomagnetic field magnitudes will provide previously unavailable precision to applications in archaeology 1 , surveying ${ }^{2}$, cardiography ${ }^{3}$ and many other fields ${ }^{4,5}$. To satisfy the needs of such differing applications magnetometers must provide, variously, high dynamic range, wide bandwidth, and high sensitivity. As an example, applications in magnetocardiography ideally require the capability of operating in Earth's field of $\sim 50 \mu \mathrm{T}$, bandwidths of DC-100 Hz, and sensitivities approaching $1 \mathrm{pT} / \sqrt{\mathrm{Hz}}^{6}$.

Superconducting quantum interference devices (SQUIDs) and fluxgates have long been established as sensitive and reliable magnetic sensors, but they have a number of limitations. SQUIDs require cryogenic cooling which limits their use as portable, compact sensors ${ }^{7}$. Fluxgates, despite their portability, lack the required sensitivity for applications with short integration times or those requiring good low frequency resolution, such as detection of rotating machinery $y^{5,8}$. Optically pumped atomic magnetometers have operating temperatures in the range $20-200^{\circ} \mathrm{C}$ and demonstrate sensitivities comparable with SQUIDs and far exceeding fluxgates. Of this class of device, spin exchange relaxation-free (SERF) atomic magnetometers lead the way in absolute sensitivity ${ }^{9}$, but their measurement range is limited to near-zero fields ${ }^{10}$. Double resonance atomic sensors have excellent dynamic range, achieving sensitivities compatible with a range of applications with the potential to operate in the Earth's field ${ }^{11}$.

In this work, we report a double-resonance atomic magnetometer that achieves $\leq 100 \mathrm{pT} / \sqrt{\mathrm{Hz}}$ sensitivity in a noisy, unshielded environment using a robust and easily-implemented feed-forward method with a $-3 \mathrm{~dB}$ cut-off frequency of $520 \mathrm{~Hz}$. The magnetometer described here uses an elliptically polarized beam to create a net magnetisation in the atomic cesium vapor due to orientation of their spins through optical pumping ${ }^{12}$. The magnetisation precesses about the ambient field, $B$, at $\omega_{L}$, the Larmor frequency such that: $\omega_{L}=\gamma B$, with proportionality constant $\gamma$ being the gyromagnetic ratio. The atoms are simultaneously optically pumped with near-resonant light and interrogated magnetically with a

a)Electronic mail: carolyn.odwyer@strath.ac.uk near-resonant oscillating magnetic field in a process known as double-resonance magnetometry ${ }^{13}$. In this case we drive the precession with a small sinusoidal RF field, $B_{R F}$. This technique has the advantage of simple geometry, with a single laser beam acting as pump and probe. Our experimental setup has been designed as a test bed for portable sensors, and we aim to minimize the complexity and number of optical elements. Alternative schemes such as amplitude or polarization modulation have additional power and space constraints due to component requirements such as acousto-optic and electrooptic modulators $^{14,15}$.

Periodic environmental magnetic noise is a challenge for unshielded magnetometers ${ }^{16,17}$. A common source of noise in unshielded indoor environments is inductively-driven magnetic fields generated at harmonics of the AC line electrical supply frequency. In our laboratory, this line noise has a typical amplitude in the $100 \mathrm{nT}$ range, observed at $50 \mathrm{~Hz}$ (United Kingdom electrical mains frequency) and higher harmonics.

Typically, environmental noise is dealt with by using passive shielding or active compensation. Passive shielding, which places the sensor inside a highly-permeable enclosure is necessarily bulky, heavy and shields the sensor from signals of interest. Active compensation, through the generation of local magnetic fields opposing components of the environmental field, can be achieved either dynamically or statically using coils and low-noise current drivers. Schemes have been implemented which modify the ambient field around the experiment in order to reduce magnetic noise ${ }^{18}$. Often an additional sensor such as a fluxgate is used to generate an error signal ${ }^{19}$. Within active compensation there are two broad categories; feedback and feed-forward. Feedback directly reacts to changes in the field based on the last instantaneous measurement ${ }^{20}$. Feed-forward takes a slice of data over time and feeds forward a prediction to the next slice. This can be implemented by feeding forward to compensation coils to cancel the ambient noise in the field external to the sensor ${ }^{21,22}$. In this work an ambient field snapshot is fed forward to the sensor itself, modifying the RF field frequency to track the ambient field more accurately as it varies in time, thus ensuring that the magnetometer operates close to its maximum sensitivity throughout the noise cycle. This can be advantageous if feedback is not implementable due to equipment latency and data transmission rate constraints.

We describe a feed-forward scheme that takes a freerunning measurement in the presence of noise and a feed for- 
ward measurement sequentially, by tracking the Larmor frequency in the same sensor. Oscillating signals of interest should not be present during the unlocked measurement. As such, the signal must be capable of being isolated, switched, or moved with respect to the sensor between the unlocked and feed-forward stage. The measurements are triggered from the AC line signal such that the feed-forward measurement phase matches the magnetic noise and the RF field frequency follows the Larmor frequency more accurately - reducing the power under the $50 \mathrm{~Hz}$ peak in the frequency domain by $22 \mathrm{~dB}$ and the overall noise floor by $20 \mathrm{~dB}$ (in the bandwidth $1 \mathrm{~Hz}$ to $1 \mathrm{kHz}$ ). Although feed-forward schemes have previously been implemented to control the ambient magnetic field, we do not know of any to date that operate in the same sensor, or by feeding forward to the RF field frequency, thereby tracking the real ambient field more closely.

\section{EXPERIMENTAL SETUP}

The experimental setup schematic of the unshielded double resonance magnetometer can be seen in Figure 1. An external cavity diode laser tuned to the cesium D1 transition is elliptically polarized and incident on a micro-fabricated vapor cell containing cesium and 700 Torr nitrogen buffer gas. The smallest inner dimension of the cell is $2 \mathrm{~mm}$. The cell is mounted on a small purpose-built printed circuit board (PCB) and heated to approximately 80 degrees using an $\mathrm{AC}$ heater driven at $17 \mathrm{MHz}$. This temperature ensures sufficient vapor pressure and thus atomic density of cesium in the cell. The PCB has an integrated RF coil that is used to apply RF fields to the cell. This is controlled in software via a digital to analog converter (DAC).

The cell is at the centre of a three-axis Helmholtz coil set which act to compensate the Earth's field and apply arbitrary fields in any orientation ${ }^{23}$. The laser light interacts with the atoms and is subsequently analysed by a half-wave plate. A Wollaston prism (WP) separates the light into its orthogonal components and directs them onto a two-channel differential photodiode. The polarization rotation due to circular dichro-

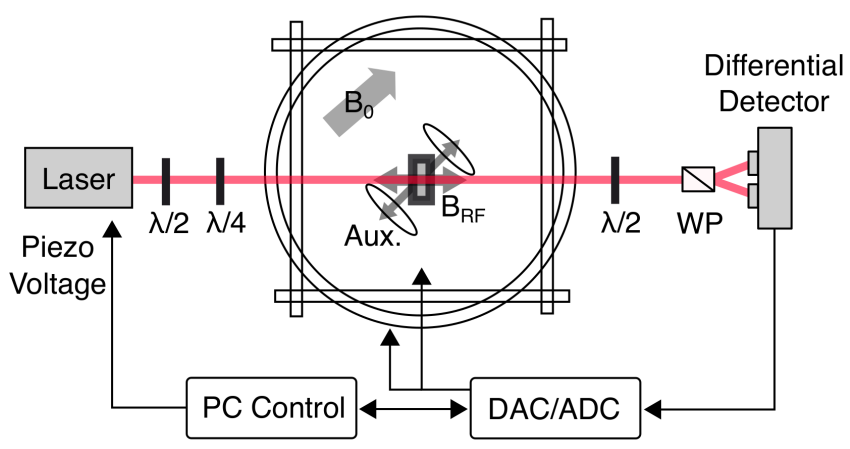

FIG. 1. Schematic of the experimental setup. The laser is elliptically polarized and incident on a microfabricated atomic cell. The ambient field, $B_{0}$ is controlled by three-axis Helmholtz coils and $B_{R F}$ is applied on a small coil mounted close to the cell. The magnetic signal is read using a balanced photodetector and both analysis and system control is done in software.(DAC: Digital to Analog Converter; ADC: Analog to Digital Converter; WP: Wollaston Prism; Aux.: Auxiliary coil) ism is read by the computer via an analog to digital converter (ADC), and demodulated at the applied $B_{R F}$ field frequency.

\section{RESULTS}

The feed forward measurement scheme comprises three distinct steps; a resonance sweep, an unlocked noise measurement, and a feed forward measurement.

The resonance sweep is generated by scanning a $300 \mathrm{nT}$ sinusoidal field, amplitude $B_{R F}$ and frequency $\omega_{R F}$ through a range of frequencies in the region of the Larmor frequency and the resultant signal is demodulated at the applied frequencies. This produces a Lorentzian resonant response, as seen in Figure 2 , with the zero-crossing corresponding to $\omega_{L}$. By fitting to the in-phase $(Y)$, quadrature $(X)$ and phase components of the demodulated signal the parameters $\Gamma$, the relaxation rate; $\omega_{L}$, the Larmor frequency; and $A$, the on-resonance amplitude can be calculated. The fit functions are as follows:

$$
\begin{aligned}
& X=\frac{x A}{1+S^{2}+x^{2}}, \\
& Y=\frac{(1+x) A}{1+S^{2}+x^{2}}
\end{aligned}
$$

where $x=\frac{\omega_{L}-\omega_{R F}}{\Gamma}, S=\frac{\Omega}{\Gamma}$, and $\Omega$ is the magnetic Rabi frequency. Parameters relevant to the feed-forward scheme are tabulated in Table I. A pre-trigger time is included before the $B_{R F}$ is applied, during which only the static field is applied. The noise on this measurement is calculated by taking the RMS deviation of the polarimeter signal while no $B_{R F}$ is applied for the same sample time as the resonance scan in question.

The measured Larmor frequency, $\omega_{L}$, is then used in a freerunning, or 'unlocked' measurement. The start of this measurement is triggered from the AC line. A constant RF field at $\omega_{L}$ is applied for a period of time and the response of the magnetometer measured. The polarimeter signal is demodulated at $\omega_{L}$ and the in-phase signal component $(X)$ is converted to

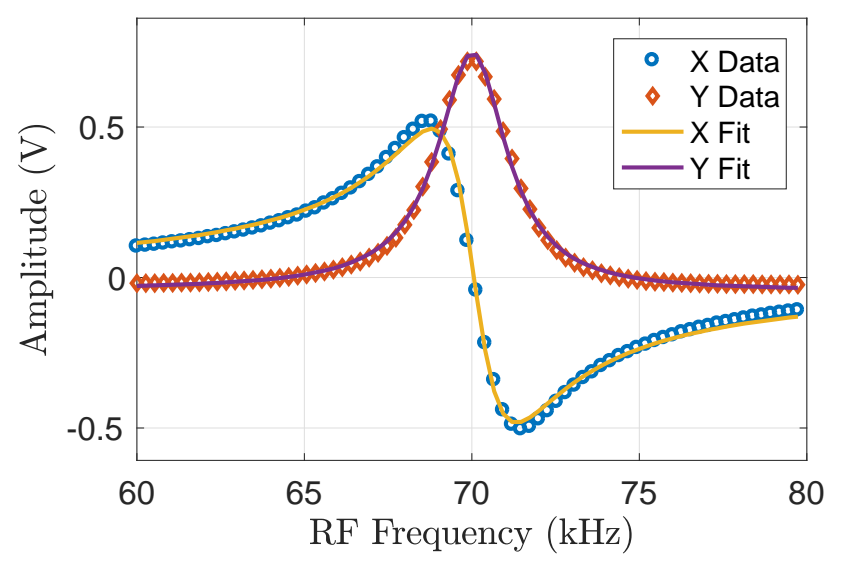

FIG. 2. In-phase and quadrature components of the magnetic resonance signal for an applied RF magnetic field that is swept through the Larmor frequency. 


\begin{tabular}{c|c}
\hline Larmor frequency, $\omega_{L}(\mathrm{kHz})$ & $70.049(11)$ \\
SNR & $4370(62)$ \\
Relaxation Rate, $\Gamma(\mathrm{kHz})$ & $1.016(14)$ \\
Sensitivity, $\sqrt{P S D}(\mathrm{pT} / \sqrt{\mathrm{Hz}})$ & $2.913(69)$ \\
\hline
\end{tabular}

TABLE I. Values of experimentally-relevant parameters derived from fits to the resonant response in Figure 2.

a magnetic field deviation using the fitted on-resonance gradient. This is a snapshot of the ambient periodic magnetic noise.

The in-phase response of the magnetometer is applied to the next measurement as a modulation of the applied RF field frequency. This measurement is also AC-line triggered in order to ensure that its phase matches the previous measurement. The sensor has been found to more accurately track the ambient field when operating in this mode. The reduction of the noise amplitude maintains the magnetic signal in the desired linear operating regime. This can be seen clearly in the demodulated data in Figure 3. The RF frequency more closely tracks the Larmor frequency on the feed-forward measurement, resulting in the peak to peak amplitude being reduced by $\sim 200 \mathrm{nT}$.

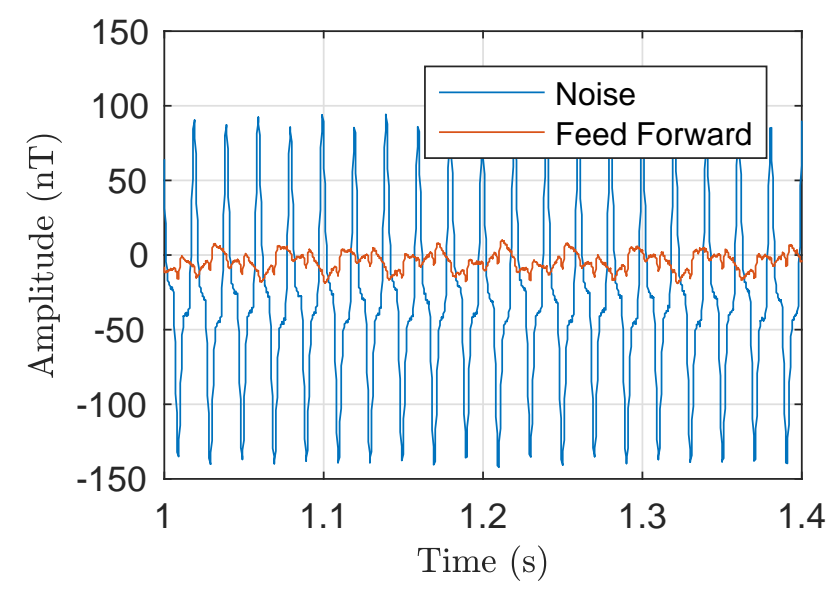

FIG. 3. Segment of demodulated magnetic field data from a 6 second period in the unlocked (blue) and feed-forward (red) modes.

The noise reduction can be seen more clearly in the frequency spectrum, as seen in Figure 4. The power under the $50 \mathrm{~Hz}$ peak is reduced by $22 \mathrm{~dB}$ in the range between 45 and $55 \mathrm{~Hz}$ as well as the peak amplitude being reduced by a factor of over 500 . The peak at $100 \mathrm{~Hz}$ is reduced by $3 \mathrm{~dB}$ between 95 and $105 \mathrm{~Hz}$, and the $150 \mathrm{~Hz}$ peak is suppressed by $21 \mathrm{~dB}$ between 145 and $155 \mathrm{~Hz}$.

The peak at $22 \mathrm{~Hz}$ corresponds to building air conditioning units which are not in phase with the AC line. These units are directly adjacent to the lab and therefore the magnetic signal is large with respect to other noise sources. The feed-forward routine does not suppress the noise from this source, and instead the noise at $22 \mathrm{~Hz}$ is increased. This will be the case with any signal not phase-locked to the mains signal present during the initial unlocked measurement. This observation highlights a limitation of our technique.

In order to test the noise cancellation technique in the presence of an arbitrary magnetic field signal, a small auxiliary Helmholtz coil pair is placed around the cell. The
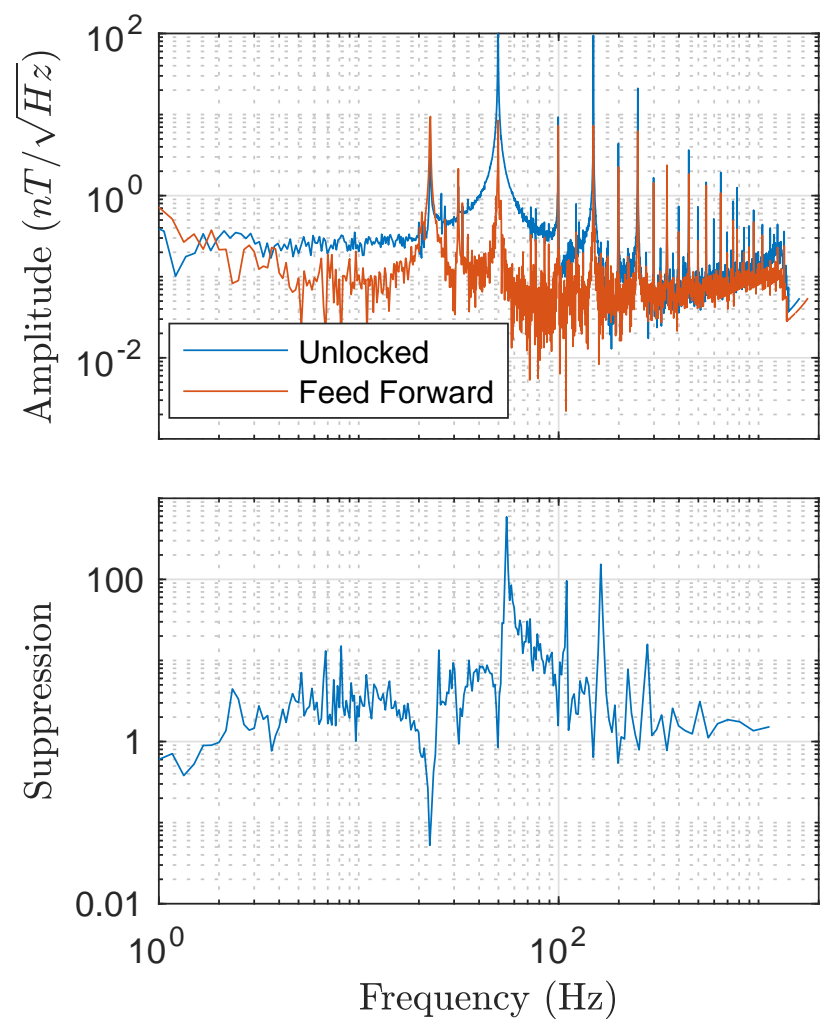

FIG. 4. Top; magnetic noise spectra for the unlocked (blue) and freerunning (red) modes. The power under the $50 \mathrm{~Hz}$ line and its harmonics can be seen to be reduced in the feed-forward mode. These data have been rescaled by the response of the sensor. Bottom; ratio of unlocked spectral response to that of feed-forward, showing ability of the feed forward technique to suppress in-phase periodic noise across the a range of frequencies. In particular the peak at $50 \mathrm{~Hz}$ is suppressed by a factor of 500. Data have been rebinned into 500 logarithmically spaced bins for clarity.

coils are aligned to the axis of maximum sensitivity of the magnetometer ${ }^{24}$. A function generator can apply oscillating currents to the coil at arbitrary frequencies during the feed forward measurement stage and this can be demodulated in software to recover the amplitude of the field.

Applying a known, constant amplitude oscillating field at different frequencies yields the frequency response of the sensor in Figure 5 from which the bandwidth of the sensor can be inferred to be $520 \mathrm{~Hz}$ (-3 dB cut-off). The region up to $100 \mathrm{~Hz}$ is flat, and we are able to resolve frequencies close to the $50 \mathrm{~Hz}$ line. This demonstrates part of this scheme's advantage over a notch filter, which would necessarily attenuate signals of interest in its band. It should be noted that the measured response function has been incorporated into the calculation of the magnetic noise spectrum in Figure 4.

The response of the sensor in the feed forward mode is seen to be the same as in the unlocked mode, in Figure 5. This is as expected, as the feed-forward technique should not change the response of the sensor, only allow smaller signals to be resolved. The sensitivity of the device in unlocked and feedforward modes is frequency specific. The resonance scan in Figure 2 has a sensitivity of $2.91 \mathrm{pT} / \sqrt{\mathrm{Hz}}$, where sensitivity is here estimated as the square root of the power spectral density (PSD): 


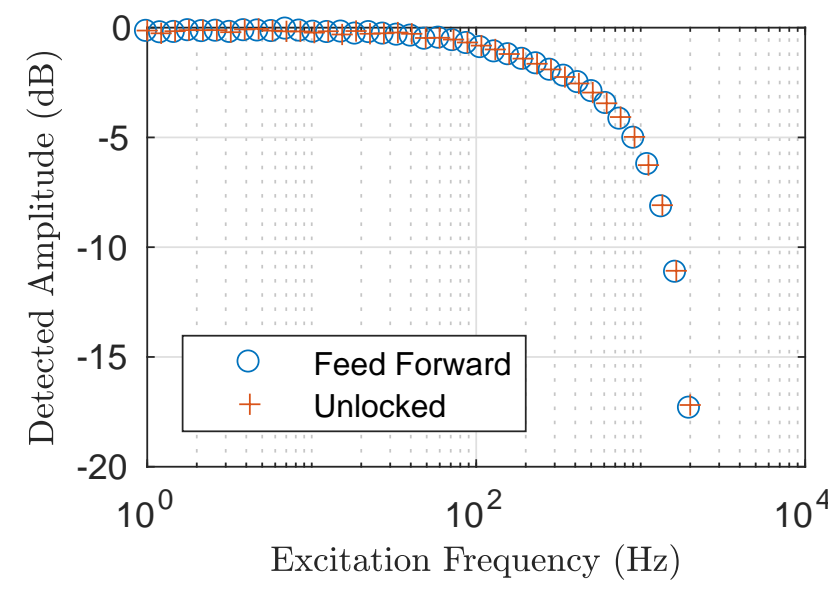

FIG. 5. Response of the sensor to applied magnetic fields of a fixed amplitude. The response rolls off to a $-3 \mathrm{~dB}$ point of $520 \mathrm{~Hz}$. The response of the sensor is identical in the unlocked and feed-forward modes.

\begin{tabular}{c|c|c}
\hline Condition & RMS Noise $(\mathrm{V})$ & $\sqrt{P S D}(\mathrm{pT} / \sqrt{\mathrm{Hz}})$ \\
\hline Laser blocked, $\mathrm{B}_{\mathrm{RF}}$ on & $1.85 \times 10^{-4}$ & $1.75(19)$ \\
Laser blocked, $\mathrm{B}_{\mathrm{RF}}$ off & $2.28 \times 10^{-4}$ & $1.46(75)$ \\
Laser on, $\mathrm{B}_{\mathrm{RF}}$ off & $2.9 \times 10^{-4}$ & $2.30(46)$ \\
Photon shot noise & $6.2 \times 10^{-6}$ & 0.05 \\
\hline
\end{tabular}

TABLE II. Contributions to noise in the sensor arising from primary noise sources in the experimental setup.

$$
\sqrt{P S D}=\frac{1}{\sqrt{B W}} \frac{\delta B}{\delta X} \delta X
$$

Where $B W$ is the measured bandwidth of the sensor, $\frac{\delta B}{\delta X}$ is the gradient on resonance and $\delta X$ is the RMS noise of the polarimeter signal after demodulation in the range $60-80 \mathrm{kHz}$, the range shown in Fig. 2. This gives an estimate of the magnetic noise the atoms are experiencing and transferring to the laser beam. This sensitivity figure reflects the ability of the sensor to resolve small changes in the field over a short measurement period of $20 \mathrm{~ms}$. Over longer measurement periods the ambient magnetic noise contributes significantly.

Table II illustrates contributions to the sensitivity of the sensor as compared to our calculated photon shot-noise sensitivity. RMS noise was recorded for each condition for a $20 \mathrm{~ms}$ period and the equivalent sensitivity calculated using (3). This gives an indication of the contribution of optical noise, RF coils and the DAQ system. This feed-forward technique has the potential to be scaled to improve the duty cycle. A shorter unlocked measurement should still yield good noise suppression for $50 \mathrm{~Hz}$ and its main harmonics, and may couple less low-frequency noise to subsequent measurements.

\section{CONCLUSION}

We have presented an experimental feed-forward technique that achieves suppression of periodic magnetic noise arising from the mains $\mathrm{AC}$ line primary frequency and its harmonics. The noise reduction is greatest around $50 \mathrm{~Hz}$ and $150 \mathrm{~Hz}, \mathrm{im}-$ proving the sensor's ability to resolve magnetic signals around this frequency band, with an amplitude reduction of 500 and a noise power reduction of $22 \mathrm{~dB}$ in the band of $\pm 5 \mathrm{~Hz}$ around the $50 \mathrm{~Hz}$ peak,. The feed-forward mode is effective in tracking more closely the ambient periodic noise, as the applied $B_{R F}$ is closer to the Larmor frequency for longer periods of time. This effect can be achieved with fast feedback, where the RF field continuously tracks the Larmor frequency in realtime. Constraints of our data acquisition system do not allow for fast enough feedback.

The response of the sensor to applied excitation fields is identical in the unlocked and feed-forward modes, rolling off to a bandwidth of $520 \mathrm{~Hz}$, which is compatible with the pressure-broadened vapor cell used. This feed-forward scheme is easily implementable in an unshielded system where large amplitude periodic noise dominates. In its current form it lends itself to applications that do not require $100 \%$ duty cycle, and those where the signal to be measured is periodic or can be deterministically turned on and off. Although the results shown here focus on mains AC noise, this technique is readily implementable in any scenario that presents periodic magnetic noise. Fetal magnetocardiography provides a possible example. A fetal magnetocardiac measurement may in theory be triggered from the mother's electrocardiogram, allowing her heart's large amplitude magnetic signal to be suppressed and improving recovery of the fetal heart rate.

\section{ACKNOWLEDGMENTS}

This work was funded by the UK Quantum Technology Hub in Sensing and Metrology, EPSRC grant № EP/M013294/1.

${ }^{1}$ E. Ben-Yosef, M. Millman, R. Shaar, L. Tauxe, and O. Lipschits, Proceedings of the National Academy of Sciences of the United States of America 114, 2160 (2017).

${ }^{2}$ D. T. Germain-Jones, Journal of Scientific Instruments 34, 1 (1957).

${ }^{3}$ J. Belfi, G. Bevilacqua, V. Biancalana, S. Cartaleva, Y. Dancheva, and L. Moi, J. Opt. Soc. Am. B 24, 2357 (2007).

${ }^{4}$ P. Bevington, R. Gartman, and W. Chalupczak, Review of Scientific Instruments 90, 13103 (2019).

${ }^{5}$ L. Marmugi, L. Gori, S. Hussain, C. Deans, and F. Renzoni, Applied Optics 56, 743 (2017).

${ }^{6}$ K. Jensen, M. A. Skarsfeldt, H. Stærkind, J. Arnbak, M. V. Balabas, S. P. Olesen, B. H. Bentzen, and E. S. Polzik, Scientific Reports 8, 16218 (2018).

${ }^{7}$ J. H. Storm, D. Drung, M. Burghoff, and R. Körber, Superconductor Science and Technology 29, 1 (2016).

${ }^{8}$ C. C. Lu, J. Huang, P. K. Chiu, S. L. Chiu, and J. T. Jeng, Sensors (Switzerland) 14, 13815 (2014).

${ }^{9}$ I. K. Kominis, T. W. Kornack, J. C. Allred, and M. V. Romalis, Nature 422, 596 (2003).

${ }^{10}$ I. M. Savukov and M. V. Romalis, Physical Review A - Atomic, Molecular, and Optical Physics 71 (2005), 10.1103/PhysRevA.71.023405.

${ }^{11}$ L. Lenci, S. Barreiro, P. Valente, H. Failache, and A. Lezama, Journal of Physics B: Atomic, Molecular and Optical Physics 45 (2012), 10.1088/0953-4075/45/21/215401.

${ }^{12}$ S. J. Ingleby, I. C. Chalmers, C. O'Dwyer, P. F. Griffin, A. S. Arnold, and E. Riis, in 2017 IEEE SENSORS (2017).

${ }^{13}$ A. L. Bloom, Appl. Opt. 1, 61 (1962).

${ }^{14}$ D. Hunter, S. Piccolomo, J. D. Pritchard, N. L. Brockie, T. E. Dyer, and E. Riis, Physical Review Applied 10, 14002 (2018).

${ }^{15}$ E. Breschi, Z. D. Grujić, P. Knowles, and A. Weis, Applied Physics Letters 104 (2014), 10.1063/1.4861458.

${ }^{16}$ J. Belfi, G. Bevilacqua, V. Biancalana, R. Cecchi, Y. Dancheva, and L. Moi, Review of Scientific Instruments 81, 065103 (2010).

${ }^{17}$ G. Bevilacqua, V. Biancalana, Y. Dancheva, and A. Vigilante, Physical Review Applied 11, 14029 (2019). 
${ }^{18}$ J. Malmivuo, J. Lekkala, P. Kontro, L. Suomaa, and H. Vihinen, Journal of Physics E: Scientific Instruments 20, 151 (1987).

${ }^{9}$ C. Deans, L. Marmugi, and F. Renzoni, Review of Scientific Instruments 89, 083111 (2018).

${ }^{20}$ H. J. M. ter Brake, R. Huonker, and H. Rogalla, Measurement Science and Technology 4, 1370 (1993).

${ }^{21} \mathrm{C}$. Flühmann and J. P. Home, Encoding a qubit in the motion of a trappedion using superpositions of displaced squeezed states presented by, Ph.D. thesis, ETH Zurich (2019)

${ }^{22}$ B. Merkel, K. Thirumalai, J. E. Tarlton, V. M. Schäfer, C. J. Ballance, T. P. Harty, and D. M. Lucas, Review of Scientific Instruments 90, 44702 (2019).

${ }^{23}$ S. J. Ingleby, P. F. Griffin, A. S. Arnold, M. Chouliara, and E. Riis, Review of Scientific Instruments 88, 043109 (2017).

${ }^{24}$ S. J. Ingleby, C. O’Dwyer, P. F. Griffin, A. S. Arnold, and E. Riis, Phys. Rev. A 96, 013429 (2017). 


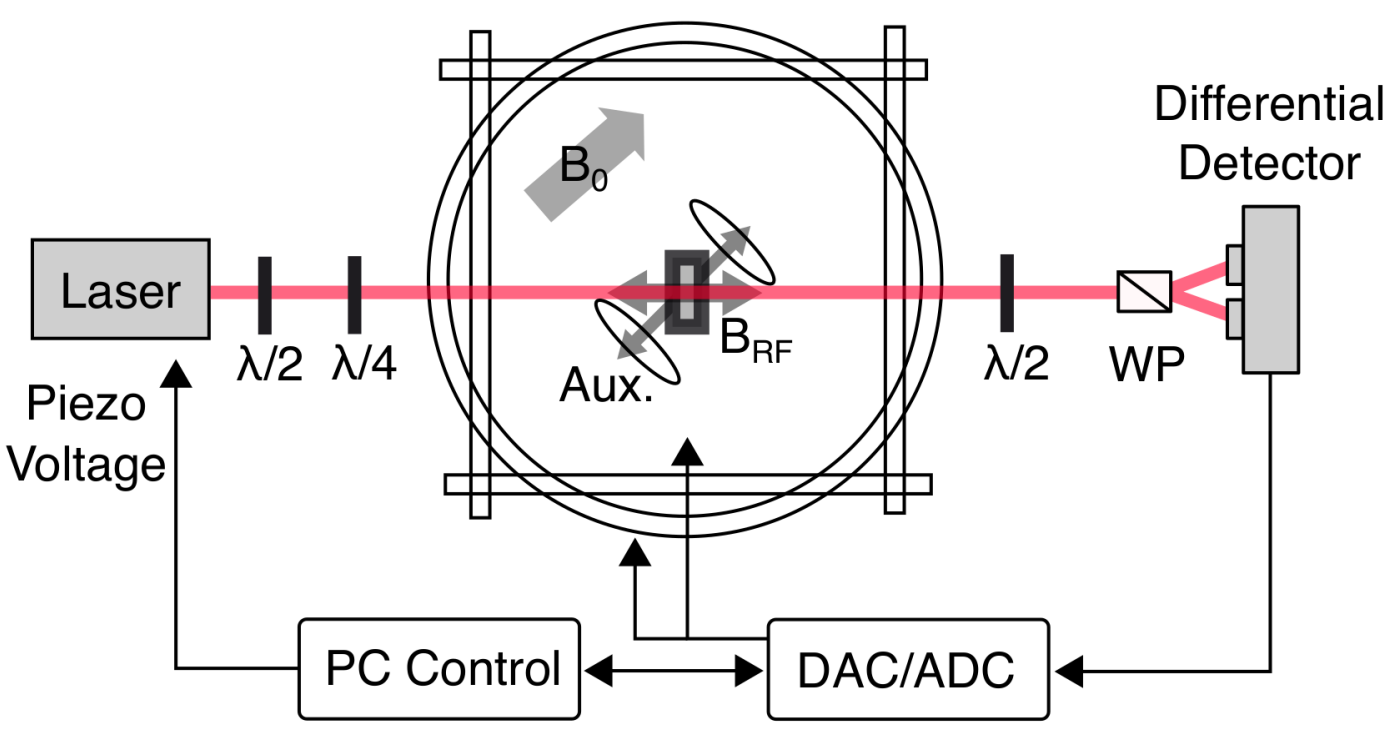




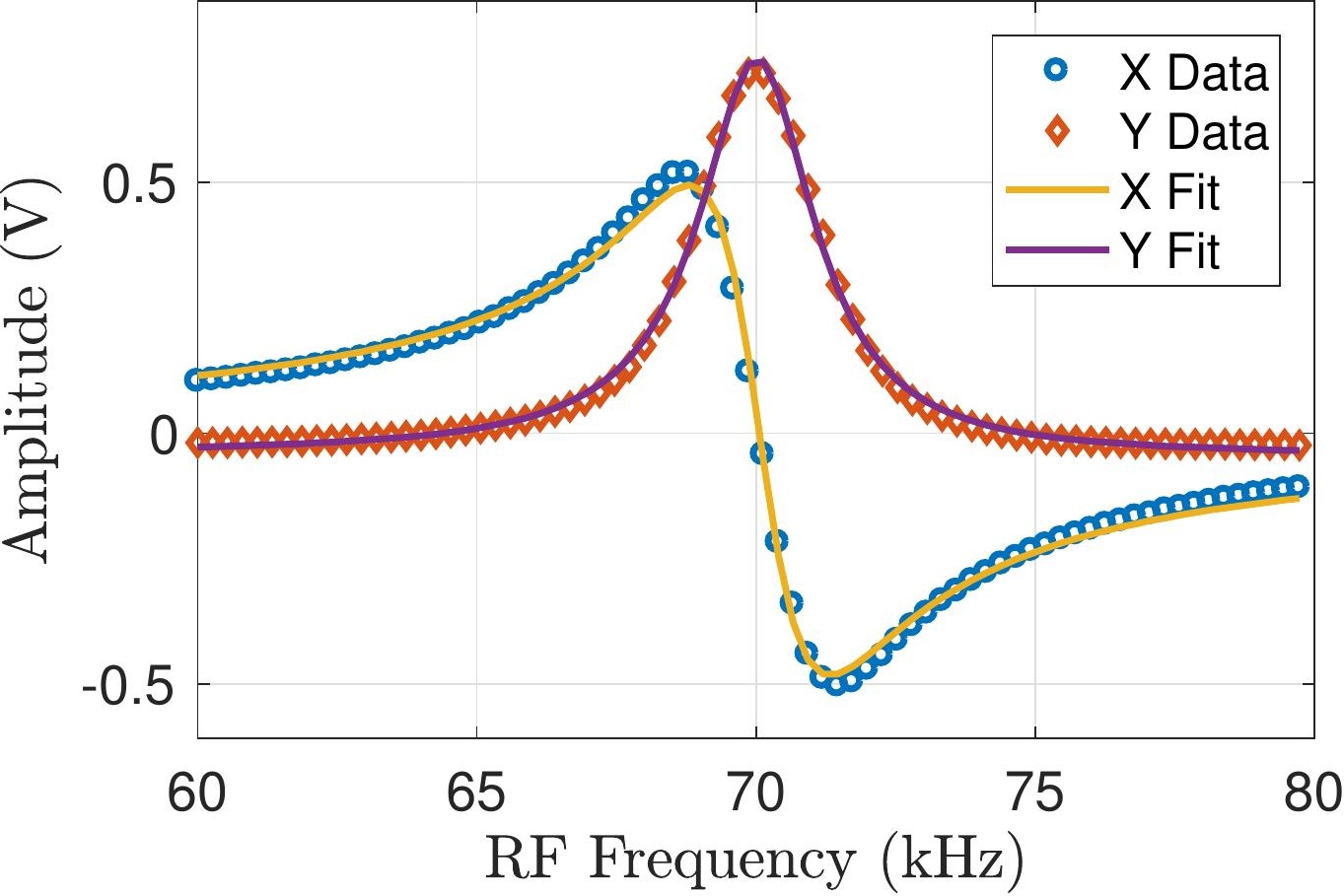




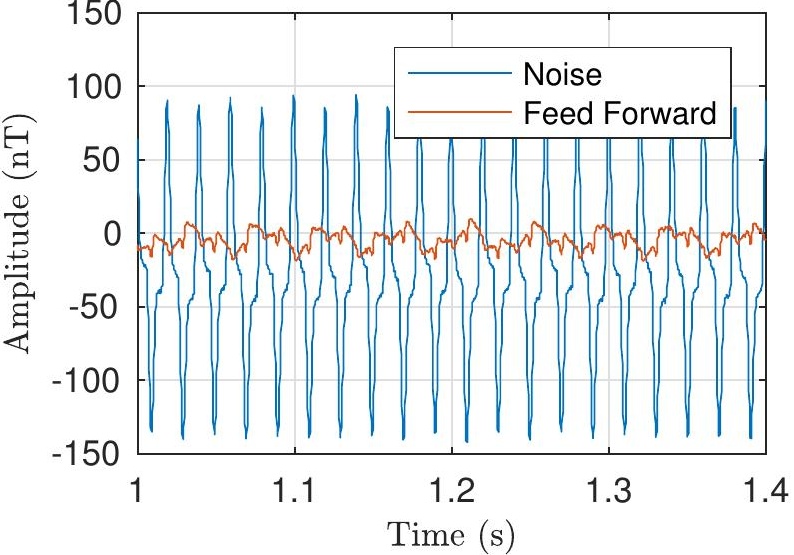



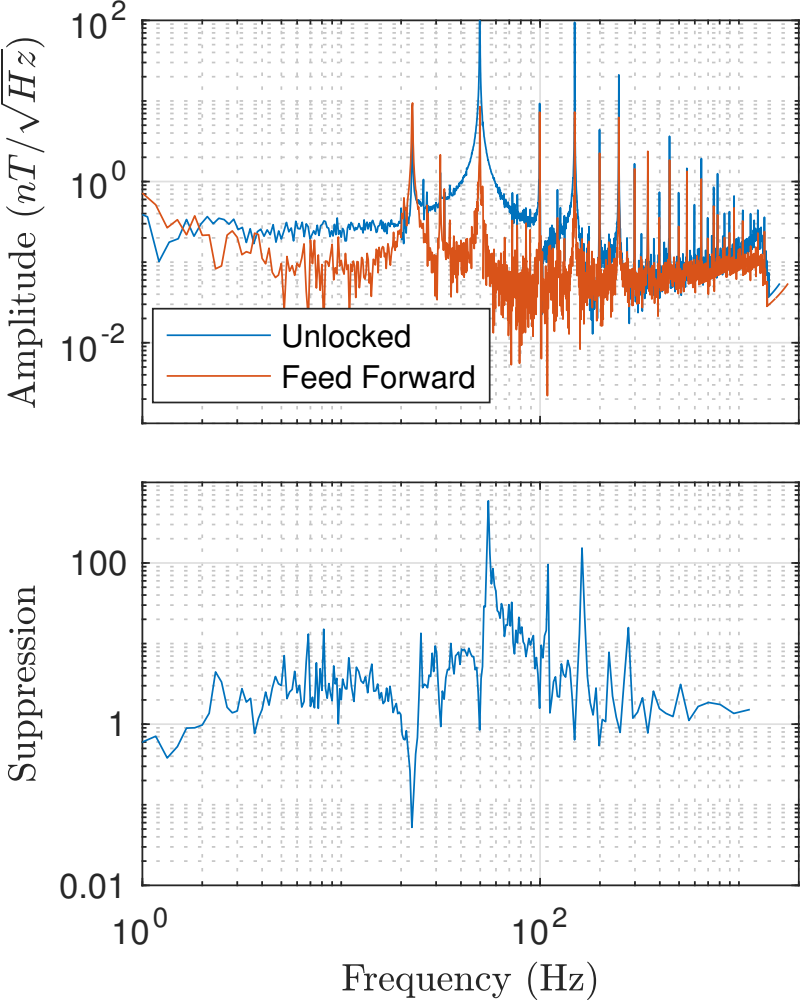


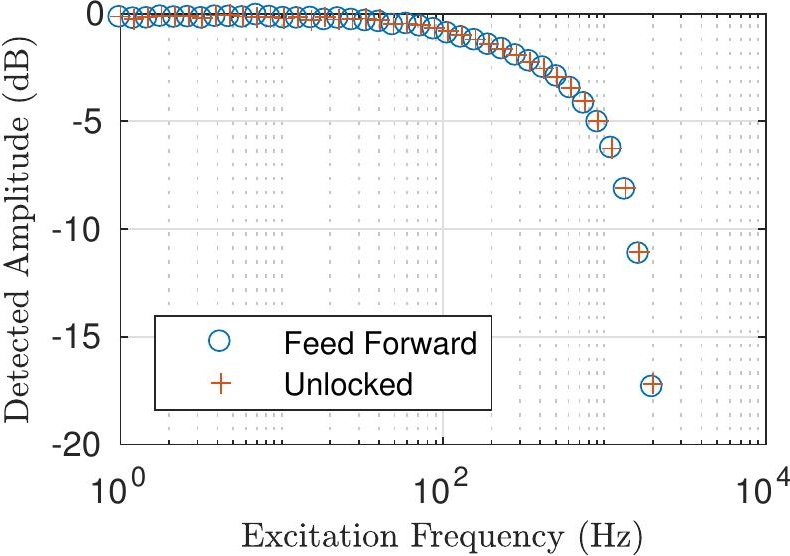

